\title{
Using Gaussian Copulas in Supervised Probabilistic Classification
}

\author{
Rogelio Salinas-Gutiérrez, Arturo Hernández-Aguirre, Mariano J. J. \\ Rivera-Meraz, and Enrique R. Villa-Diharce \\ Center for Research in Mathematics (CIMAT), Guanajuato, México \\ \{rsalinas, artha, mrivera, villadi\}@cimat.mx
}

\begin{abstract}
This chapter introduces copula functions and the use of the Gaussian copula function to model probabilistic dependencies in supervised classification tasks. A copula is a distribution function with the implicit capacity to model non linear dependencies via concordance measures, such as Kendall's $\tau$. Hence, this chapter studies the performance of a simple probabilistic classifier based on the Gaussian copula function. Without additional preprocessing of the source data, a supervised pixel classifier is tested with a 50-images benchmark; the experiments show this simple classifier has an excellent performance.
\end{abstract}

Key words: Gaussian copula, supervised classification.

\section{Introduction}

In Pattern Recognition applications many algorithms and models have been proposed for many tasks, specially for clustering, regression and classification. Applications in which a training data set with categories and attributes is available and the goal is to assign a new object to one of a finite number of discrete categories are known as supervised classification problems [2,12,15]. In this work we present the use of the Gaussian copula function as an alternative for modeling dependence structure in a supervised probabilistic classifier.

Copula functions are suitable tools in statistics for modeling multiple dependence, not necessarily linear dependence, in several random variables. For this reason, copula functions have been widely used in economics and finance [5, $7,9,25,26]$. More recently copula function have been used in other fields such as climate [22], oceanography [6], hydrology [10], geodesy [1], reliability [17], evolutionary computation [20,21] and engineering [11]. By using copula theory, a joint distribution can be built with a copula function and, possibly, several different marginal distributions. Copula theory has been used also for modeling multivariate distributions in unsupervised learning problems such as image segmentation $[4,8]$ and retrieval tasks $[16,19,24]$. In [13], the bivariate copula functions Ali-Mikhail-Haq, Clayton, Frank and Gumbel are used for unsupervised classification. These copulas are well defined for two variables but when extended to three or more variables several complications arise (for instance, 
undefined copula parameters), preventing their generalization and applicability. For the Gaussian copula however, there exist a simple "general formula" for any number of variables. This work introduces the use of Gaussian copula in supervised classification, and compares an independent probabilistic classifier with a copula-based probabilistic classifier.

The content of the chapter is the following: Section 2 is a short introduction to copula functions, Section 3 presents a copula based probabilistic model for classification. Section 4 presents the experimental setting to classify an image database, and Section 5 summarizes the conclusions.

\section{Copula Functions}

The copula concept was introduced 50 years ago by Sklar [23] to separate the effect of dependence from the effect of marginal distributions in a joint distribution. Although copula functions can model linear and nonlinear dependencies, they have been barely used in computer science applications where nonlinear dependencies are common and need to be represented.

Definition 1. A copula $C$ is a joint distribution function of standard uniform random variables. That is,

$$
C\left(u_{1}, \ldots, u_{d}\right)=P\left(U_{1} \leq u_{1}, \ldots, U_{d} \leq u_{d}\right)
$$

where $U_{i} \sim U(0,1)$ for $i=1, \ldots, d$.

For a more formal definition of copula functions, the reader is referred to [14, 18]. The following result, known as Sklar's theorem, states how a copula function is related to a joint distribution function.

Theorem 1 (Sklar's theorem). Let $F$ be a d-dimensional distribution function with marginals $F_{1}, F_{2}, \ldots, F_{d}$, then there exists a copula $C$ such that for all $x$ in $\overline{\mathbb{R}}^{d}$,

$$
F\left(x_{1}, x_{2}, \ldots, x_{d}\right)=C\left(F_{1}\left(x_{1}\right), F_{2}\left(x_{2}\right), \ldots, F_{d}\left(x_{d}\right)\right),
$$

where $\overline{\mathbb{R}}$ denotes the extended real line $[-\infty, \infty]$. If $F_{1}\left(x_{1}\right), F_{2}\left(x_{2}\right), \ldots, F_{d}\left(x_{d}\right)$ are all continuous, then $C$ is unique. Otherwise, $C$ is uniquely determined on $\operatorname{Ran}\left(F_{1}\right) \times \operatorname{Ran}\left(F_{2}\right) \times \cdots \times \operatorname{Ran}\left(F_{d}\right)$, where Ran stands for the range.

According to Theorem 1, any joint distribution function $F$ with continuous marginals $F_{1}, F_{2}, \ldots, F_{d}$ has associated a copula function $C$. Moreover, the associated copula $C$ is a function of the marginal distributions $F_{1}, F_{2}, \ldots, F_{d}$. An important consequence of Theorem 1 is that the $d$-dimensional joint density $f$ and the marginal densities $f_{1}, f_{2}, \ldots, f_{d}$ are also related:

$$
f\left(x_{1}, \ldots, x_{d}\right)=c\left(F_{1}\left(x_{1}\right), \ldots, F_{d}\left(x_{d}\right)\right) \cdot \prod_{i=1}^{d} f_{i}\left(x_{i}\right),
$$


where $c$ is the density of the copula $C$. The Equation (1) shows that the product of marginal densities and a copula density builds a $d$-dimensional joint density. Notice that the dependence structure is given by the copula function and the marginal densities can be of different distributions. This contrasts with the usual way to construct multivariate distributions, which suffers from the restriction that the marginals are usually of the same type. The separation between marginal distributions and a dependence structure explains the modeling flexibility given by copula functions.

\subsection{Gaussian Copula Function}

There are several parametric families of copula functions, such as Student's t copula and Archimedean copulas. One of these families is the Gaussian copula function.

Definition 2. The copula associated to the joint standard Gaussian distribution is called Gaussian copula.

According to Definition 2 and Theorem 1, if the $d$-dimensional distribution of a random vector $\left(Z_{1}, \ldots, Z_{d}\right)$ is a joint standard Gaussian distribution, then the associated Gaussian copula has the following expression:

$$
C\left(\Phi\left(z_{1}\right), \ldots, \Phi\left(z_{d}\right) ; \Sigma\right)=\int_{-\infty}^{z_{1}} \cdots \int_{-\infty}^{z_{d}} \frac{e^{-\frac{1}{2} t^{\prime} \Sigma^{-1} t}}{(2 \pi)^{(n / 2)}|\Sigma|^{1 / 2}} d t_{d} \cdots d t_{1}
$$

or equivalently,

$$
C\left(u_{1}, \ldots, u_{d} ; \Sigma\right)=\int_{-\infty}^{\Phi^{-1}\left(u_{1}\right)} \cdots \int_{-\infty}^{\Phi^{-1}\left(u_{d}\right)} \frac{e^{-\frac{1}{2} t^{\prime} \Sigma^{-1} t}}{(2 \pi)^{(n / 2)}|\Sigma|^{1 / 2}} d t_{d} \cdots d t_{1}
$$

where $\Phi$ is the cumulative distribution function of the marginal standard Gaussian distribution and $\Sigma$ is a symmetric matrix with main diagonal of ones. The elements outside the main diagonal of matrix $\Sigma$ are the pairwise correlations $\rho_{i j}$ between variables $Z_{i}$ and $Z_{j}$, for $i, j=1, \ldots, d$ and $i \neq j$. It can be noticed that a $d$-dimensional standard Gaussian distribution has mean vector zero and a correlation matrix $\Sigma$ with $d(d-1) / 2$ parameters.

The dependence parameters $\rho_{i j}$ of a $d$-dimensional Gaussian copula can be estimated using the maximum likelihood method. To do so, we follow the steps of Algorithm 1.

Due to Equation (1), the $d$-dimensional Gaussian copula density can be calculated as:

$$
\begin{aligned}
c\left(\Phi\left(z_{1}\right), \ldots, \Phi\left(z_{d}\right) ; \Sigma\right) & =\frac{\frac{1}{(2 \pi)^{(d / 2)}|\Sigma|^{1 / 2}} e^{-\frac{1}{2} z^{\prime} \Sigma^{-1} z}}{\prod_{i=1}^{d} \frac{1}{(2 \pi)^{1 / 2}} e^{-\frac{1}{2} z_{i}^{2}}} \\
& =\frac{1}{|\Sigma|^{1 / 2}} e^{-\frac{1}{2} z^{\prime}\left(\Sigma^{-1}-I\right) z}
\end{aligned}
$$




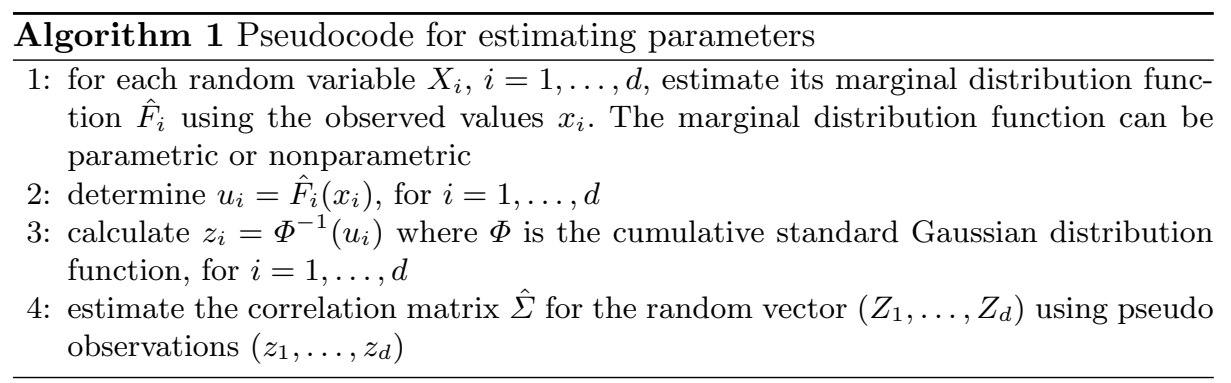

Given that a Gaussian copula is a distribution function it is possible to simulate data from it. The main steps are the following: once a correlation matrix $\Sigma$ is specified, a data set can be generated from a joint standard Gaussian distribution. The next step consists of transforming this data set using the cumulative distribution function $\Phi$. For random vectors with a Gaussian copula associated to their joint distribution, the first step is to generate data from the copula and then determining their quantiles by means of their cumulative distribution functions. Algorithm 2 and Figures 1, 2 and 3 illustrate the sampling procedure for different correlations.

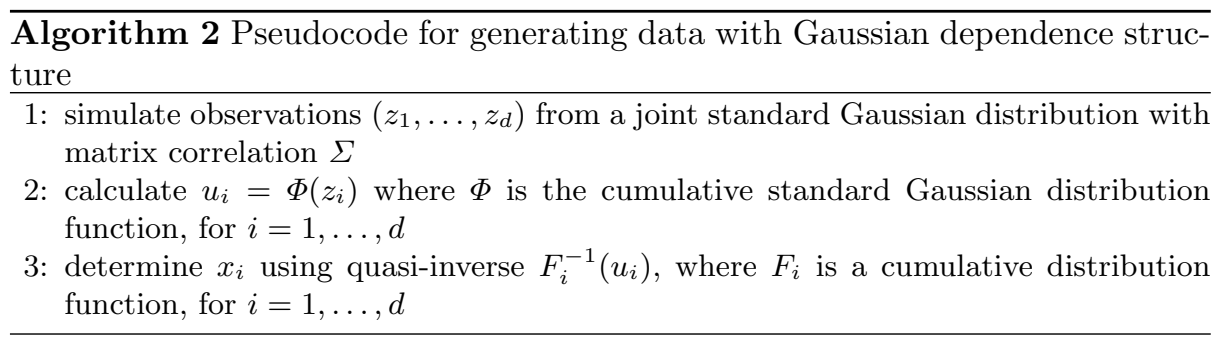

Figure 1-(a) shows 500 bivariate data with correlation $\rho=-0.5$ drawn from a bivariate standard Gaussian distribution (step 1, Algorithm 2). The histogram on the vertical axis and the histogram on the horizontal axis illustrate that both marginals are univariate standard Gaussian distributions. This data set is used to obtain a sample from a Gaussian copula, as shown in Figure 1-(b) (step 2, Algorithm 2). Both histograms illustrate that marginals are uniform, according to Definition 1. Figure 1-(c) shows a sample from a joint distribution with Gaussian copula and Beta marginals (step 3, Algorithm 2). This sample is obtained using the data set of Figure 1-(b). Figure 1-(d) shows a sample from a joint distribution with Gaussian copula, Student's t marginal distribution and exponential marginal distribution (step 3, Algorithm 2). This sample is also obtained from the data set of Figure 1-(b). In order to appreciate how the correlation parameter modifies the dependence structure, Figures 2 and 3 show the same information as Figure 1 with $\rho=-0.7$ and $\rho=-0.95$, respectively. 


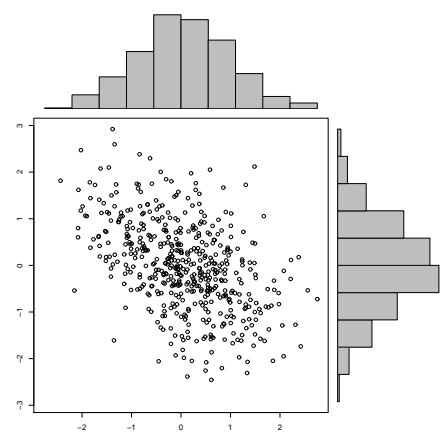

(a)

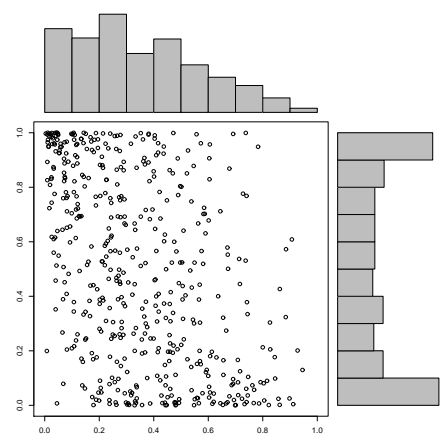

(c)

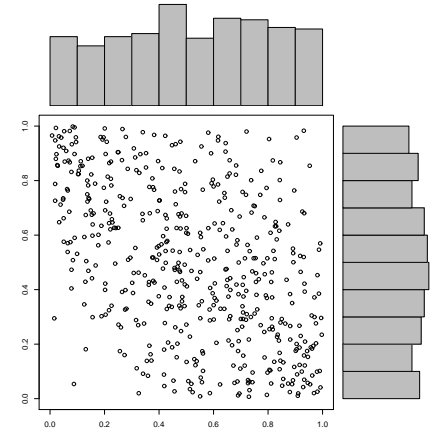

(b)

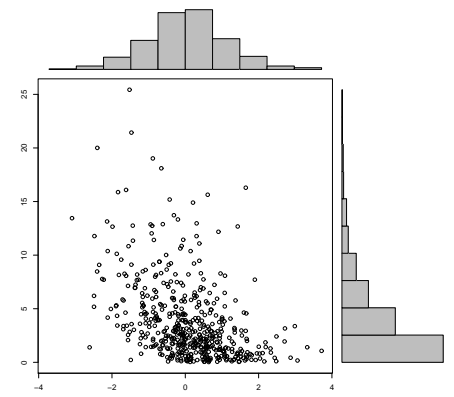

(d)

Fig. 1. (a) A sample of 500 points from a standard Gaussian distribution with parameter $\rho=-0.50$. (b) The corresponding sample for a Gaussian copula. (c) The associated sample for a joint distribution with marginal Beta distributions with parameters $(1,2)$ (histogram on the horizontal axis) and $(0.5,0.5)$ (histogram on the vertical axis). (d) The associated sample for a joint distribution with marginal t-Student distribution with 8 degrees of freedom (histogram on the horizontal axis) and marginal exponential distribution with mean 4 (histogram on the vertical axis). 


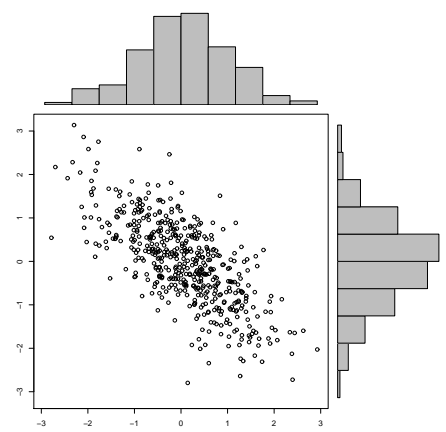

(a)

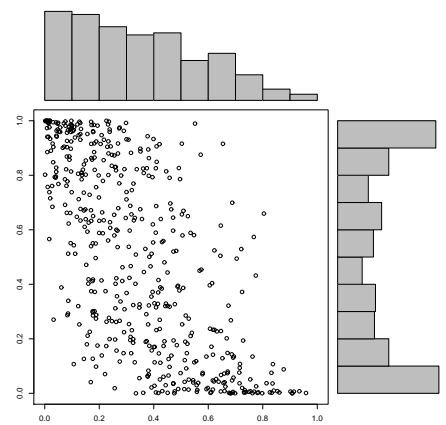

(c)

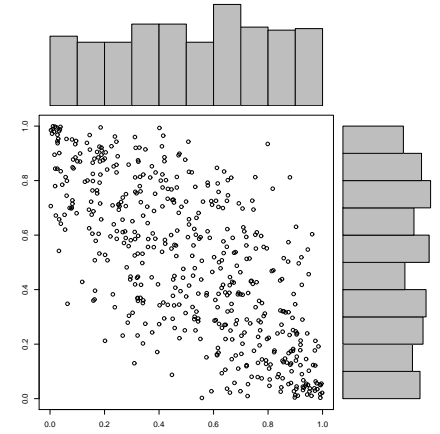

(b)

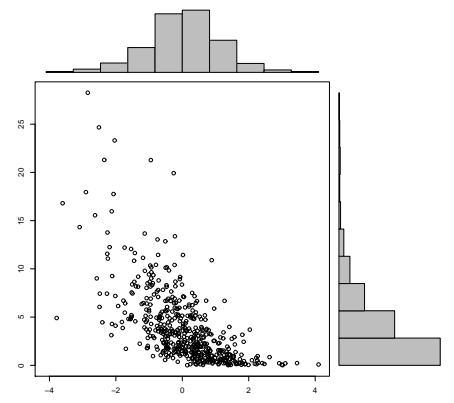

(d)

Fig. 2. (a) A sample of 500 points from a standard Gaussian distribution with parameter $\rho=-0.70$. (b) The corresponding sample for a Gaussian copula. (c) The associated sample for a joint distribution with marginal Beta distributions with parameters $(1,2)$ (histogram on the horizontal axis) and $(0.5,0.5)$ (histogram on the vertical axis). (d) The associated sample for a joint distribution with marginal t-Student distribution with 8 degrees of freedom (histogram on the horizontal axis) and marginal exponential distribution with mean 4 (histogram on the vertical axis). 


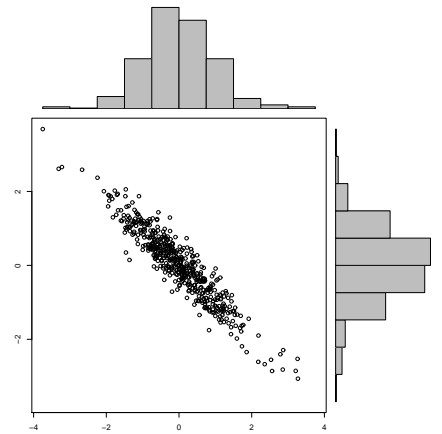

(a)

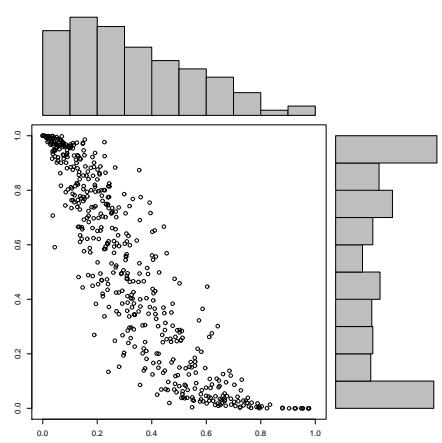

(c)

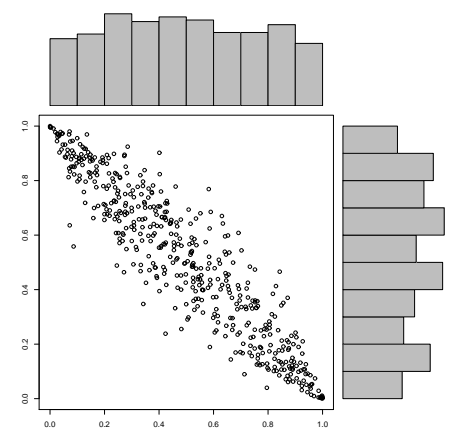

(b)

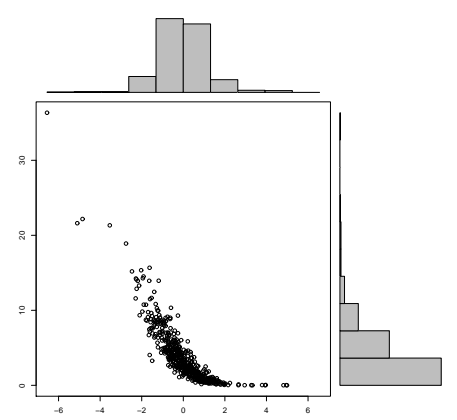

(d)

Fig. 3. (a) A sample of 500 points from a standard Gaussian distribution with parameter $\rho=-0.95$. (b) The corresponding sample for a Gaussian copula. (c) The associated sample for a joint distribution with marginal Beta distributions with parameters $(1,2)$ (histogram on the horizontal axis) and $(0.5,0.5)$ (histogram on the vertical axis). (d) The associated sample for a joint distribution with marginal t-Student distribution with 8 degrees of freedom (histogram on the horizontal axis) and marginal exponential distribution with mean 4 (histogram on the vertical axis). 
Although the correlation is used to generate data from a Gaussian copula, it is not necessary the same for joint distributions with Gaussian copula and non-Gaussian marginals. However, the data sets in Figure 1 have the same concordance value measured in Kendall's $\tau$. This important result for parametric bivariate copulas (see [18]) is explained through the equation:

$$
\tau\left(X_{1}, X_{2}\right)=4 \int_{0}^{1} \int_{0}^{1} C\left(u_{1}, u_{2} ; \theta\right) d C\left(u_{1}, u_{2} ; \theta\right)-1
$$

which relates the dependence parameter $\theta$ of a copula and Kendall's $\tau$. For a bivariate Gaussian copula, Equation (3) can be written as

$$
\tau=\frac{2}{\pi} \arcsin (\rho)
$$

Given that is well established how to estimate correlation matrixes, evaluate densities, and calculate integrals for the multidimensional Gaussian distribution, the Gaussian copula function is relatively easy to implement.

\section{The Probabilistic Classifier}

As noted, the aim of this work is to introduce the use of Gaussian copula functions in supervised classification. According to Theorem 1, we can employ a copula function in a probabilistic classifier, such as a Bayessian classifier. In this section we present a three dimensional probabilistic model based on three empirical distribution functions and a trivariate dimensional Gaussian copula function.

The Bayes' theorem states the following:

$$
P(K=k \mid E=e)=\frac{P(E=e \mid K=k) \times P(K=k)}{P(E=e)},
$$

where $P(K=k \mid E=e)$ is the posterior probability, $P(E=e \mid K=k)$ is the likelihood function, $P(K=k)$ is the prior probability and $P(E=e)$ is the data probability.

The Equation (5) has been used as a tool in supervised classification. A probabilistic classifier can be designed comparing the posterior probability that an object belongs to class $K$ given its attributes $E$. The object is then assigned to the class with the highest posterior probability. For practical reasons, the data probability $P(E)$ does not need to be evaluated for comparing posterior probabilities. Furthermore, the prior probability $P(K)$ can be substituted by an uniform distribution if the user does not have an informative distribution.

\subsection{The Probabilistic Classifier based on Gaussian Copula Function}

For continuous attributes, a Gaussian copula function can be used for modeling the dependence structure in the likelihood function. In this case, the Bayes' 
theorem can be written as:

$$
P(K=k \mid e)=\frac{c\left(F_{1}\left(e_{1}\right), \ldots, F_{n}\left(e_{n}\right) \mid k, \Sigma\right) \times \prod_{i=1}^{n} f_{i}\left(e_{i} \mid k\right) \times P(K=k)}{f\left(e_{1}, \ldots, e_{n}\right)},
$$

where $F_{i}$ and $f_{i}$ are the marginal distribution functions and the marginal densities of attributes, respectively. The function $c$ is a $d$-dimensional Gaussian copula density defined by Equation (2). As can be seen in Equation (6), each category determines a likelihood function.

\subsection{The Probabilistic Classifier based on Independent Model}

By considering conditional independence among the attributes in Equation (6), or equivalently, an independent structure in the likelihood function given a category, a probabilistic classifier can use the following expression in order to calculate posterior probabilities:

$$
P(K=k \mid e)=\frac{\prod_{i=1}^{n} f_{i}\left(e_{i} \mid k\right) \times P(K=k)}{f\left(e_{1}, \ldots, e_{n}\right)} .
$$

Equation (7) uses an independent structure given by a copula density equals to one. This independent copula density can be also obtained by a Gaussian copula function when matrix $\Sigma$ is the identity matrix $I$.

\subsection{An Application Example}

Consider the following specific classification problem: assign a pixel to a certain class according to its color attributes. If we have information about the color distribution of each class, then we can use this information and the Bayes' theorem in order to classify new pixels. This is an example of supervised classification. For a red-green-blue (RGB) color space and two classes, a Gaussian copula based classifier can be written as

$$
P(k \mid r, g, b)=\frac{c\left(F_{R}(r), F_{G}(g), F_{B}(b) \mid k, \Sigma\right) f_{R}(r \mid k) f_{G}(g \mid k) f_{B}(b \mid k) \times P(k)}{f(r, g, b)},
$$

where $c$ is a trivariate Gaussian copula density.

In order to classify a pixel, we use in Equation (8) a prior probability $P(K=$ $k$ ) based on the uniform distribution, nonparametric marginal densities $\hat{f}$ based on histograms to approximate $f_{R}(r \mid k), f_{G}(g \mid k)$ and $f_{B}(b \mid k)$, and nonparametric marginal distributions $\hat{F}$ based on empirical cumulative distribution functions to approximate $F_{R}(r), F_{G}(g)$ and $F_{B}(b)$. For modeling the dependence structure of the likelihood function $f(r, g, b \mid k)$ we present the use of a trivariate Gaussian copula function. 


\section{Experiments}

We use two probabilistic models in order to classify pixels of 50 test images. The first model is an independent probabilistic model (I-M) based on the product of marginal distributions. The second model is a copula-based model (GC-M) that takes into account a dependence structure by means of a trivariate Gaussian copula. The image database was used in [3] and is available online [27]. This image database provides information about two classes: the foreground and the background. The training data and the test data are contained in the labelling-lasso files [27], whereas the correct classification is contained in the segmentation files. Figures 4, 5 and 6 show the description of three images from the database. Table 3 shows a description for each image. Although the database is used for segmentation purposes, the aim of this work is to introduce the use of the Gaussian copula function in supervised color pixel classification. We use the information for supervised color pixel classification, without taking into account the spatial information.

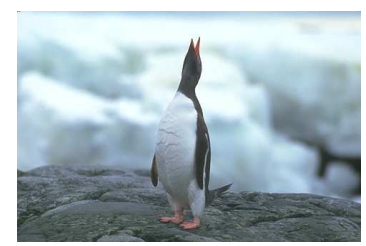

(a)

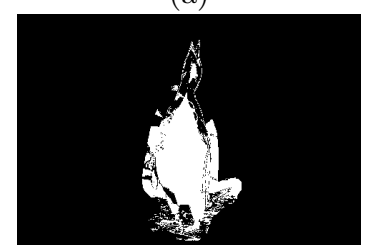

(d)

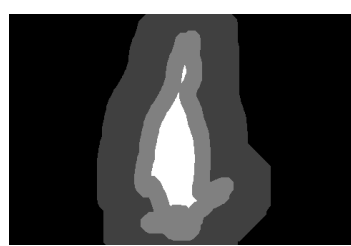

(b)

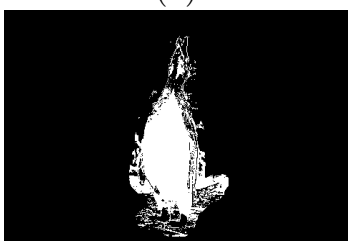

(e)

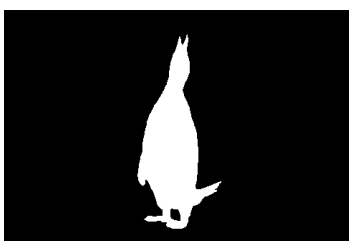

(c)

Fig. 4. (a) The color image. (b) The labelling-lasso image with the training data for background (dark gray), for foreground (white) and the test data (gray). (c) The correct classification with foreground (white) and background (black). (d) Classification made by I-M. (e) Classification made by GC-M.

Two evaluation measures are used in this work: accuracy and Tanimoto coefficient. The accuracy is described in Figure 7. We define the positive class as foreground and the negative class as background.

The Tanimoto coefficient (TC) is also known as Jaccard similarity measure. This measure, TC, is defined as:

$$
T C(k)=\frac{V_{m \cap g}(k)}{V_{m \cup g}(k)}
$$




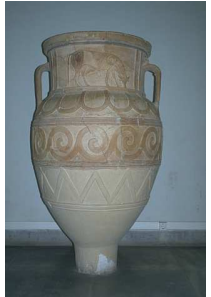

(a)

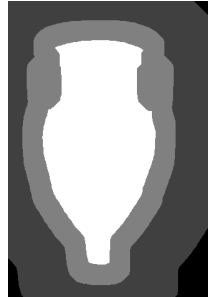

(b)

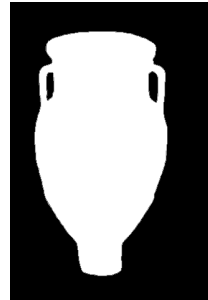

(c)

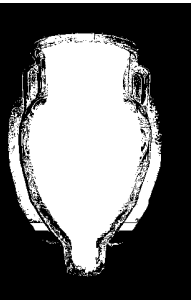

(d)

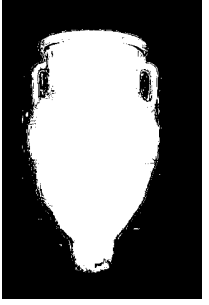

(e)

Fig. 5. (a) The color image. (b) The labelling-lasso image with the training data for background (dark gray), for foreground (white) and the test data (gray). (c) The correct classification with foreground (white) and background (black). (d) Classification made by I-M. (e) Classification made by GC-M.

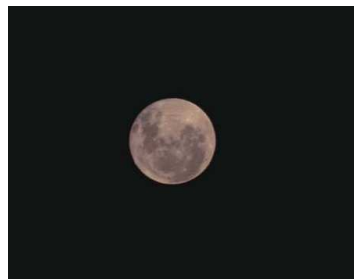

(a)

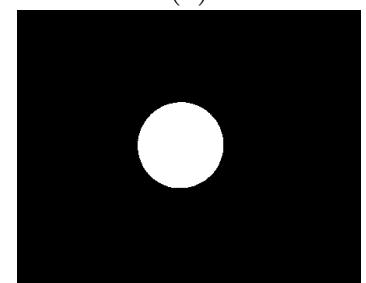

(d)

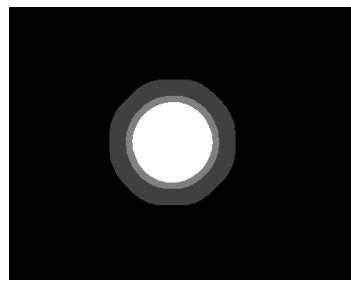

(b)

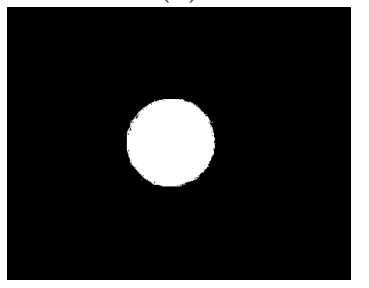

(e)

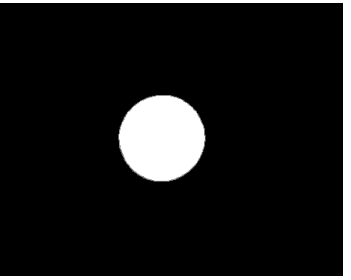

(c)

Fig. 6. (a) The color image. (b) The labelling-lasso image with the training data for background (dark gray), for foreground (white) and the test data (gray). (c) The correct classification with foreground (white) and background (black). (d) Classification made by I-M. (e) Classification made by GC-M.

\begin{tabular}{|r|c|c|}
\hline & \multicolumn{2}{|c|}{$\begin{array}{c}\text { Truth } \\
\text { Positive }\end{array}$} \\
Negative \\
\hline Model Positive & $t p$ & $f p$ \\
\hline Negative & $f n$ & $t n$ \\
\hline
\end{tabular}

(a)

$$
\text { accuracy }=\frac{t p+t n}{t p+f p+f n+t n}
$$

(b)

Fig. 7. (a) A confusion matrix for binary classification, where $t p$ are true positive, $f p$ false positive, $f n$ false negative, and $t n$ true negative counts. (b) Definition of accuracy used in this work. 
where $V_{m \cap g}(k)$ denotes the number of pixels classified as class $k$ by both the model and the ground truth and $V_{m \cup g}(k)$ denotes the number of pixels classified as class $k$ by either the model or the ground truth.

\subsection{Numerical Results}

In Table 1 we summarize the measure values reached by the independent probabilistic model (I-M) and the copula-based model (GC-M). The information about the number of pixels well classified for each class is reported in Table 3. We include in Table 4 the performances of I-M and GC-M for each image.

Table 1. Descriptive results for all evaluation measures. BG stands for the background class and FG stands for the foreground class.

\begin{tabular}{|c|c|c|c|c|c|}
\hline Measure & Minimum & Median & Mean & Maximum & Std. deviation \\
\hline \multicolumn{5}{|c|}{ I-M } \\
\hline Tanimoto coefficient - BG & 0.369 & 0.690 & 0.695 & 0.955 & 0.143 \\
\hline Tanimoto coefficient - FG & 0.341 & 0.633 & 0.638 & 0.953 & 0.168 \\
\hline Accuracy & 0.571 & 0.792 & 0.795 & 0.976 & 0.107 \\
\hline \multicolumn{5}{|c|}{ GC-M } \\
\hline Tanimoto coefficient - BG & 0.450 & 0.816 & 0.797 & 0.976 & 0.118 \\
\hline Tanimoto coefficient - FG & 0.375 & 0.780 & 0.758 & 0.972 & 0.141 \\
\hline Accuracy & 0.587 & 0.889 & 0.871 & 0.987 & 0.083 \\
\hline
\end{tabular}

To properly compare the performance of the probabilistic models, we conducted a hypothesis test based on a Bootstrap method for the differences between the means of accuracy and Tanimoto coefficients, for both probabilistic models. Table 2 shows the confidence interval for the means, and the corresponding pvalue.

Table 2. Results for the difference between evaluation measure means in each model. A 95\% confidence interval and a p-value are obtained through a Bootstrap technique. $\mathrm{BG}$ stands for the background class and FG stands for the foreground class.

\begin{tabular}{|c|c|c|c|}
\hline Measure & \multicolumn{2}{|c|}{$95 \%$ Interval } & p-value \\
\hline Tanimoto coefficient - BG & $-1.52 \mathrm{E}-01$ & $-5.18 \mathrm{E}-02$ & $2.67 \mathrm{E}-04$ \\
\hline Tanimoto coefficient - FG & $-1.80 \mathrm{E}-01$ & $-5.96 \mathrm{E}-02$ & $3.67 \mathrm{E}-04$ \\
\hline Accuracy & $-1.14 \mathrm{E}-01$ & $-3.94 \mathrm{E}-02$ & $2.67 \mathrm{E}-04$ \\
\hline
\end{tabular}

\subsection{Discussion}

According to Table 1, the GC-M shows the best behaviour for all evaluation measures. For instance, the mean accuracy for the I-M, 79.5\%, is less than the 
mean accuracy for the GC-M, 87.1\%. This means that using a I-M approximately has $8 \%$ more error rate than using a GC-M.

The average of the Tanimoto coefficient for the background class is greater than the average of the Tanimoto coefficient for the foreground class, for both I-M and GC-M (see Table 1). These coefficients are shown for each image in Figure 8-(a) and Figure 8-(b). Notice the Tanimoto coefficients for GC-M on the background and foreground are very similar, denoting a better classification than I-M. In most of the images and for each class, we can see in Figure 8-(c) and 8-(d) (also in Table 4) that GC-M outperforms I-M. In average, according to Table 1, the GC-M improves the I-M in both classes. For the foreground class from $63.8 \%$ to $75.8 \%$, and for the background class from $69.5 \%$ to $79.7 \%$.

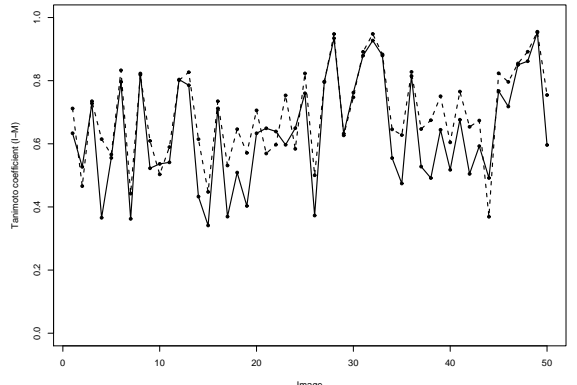

(a)

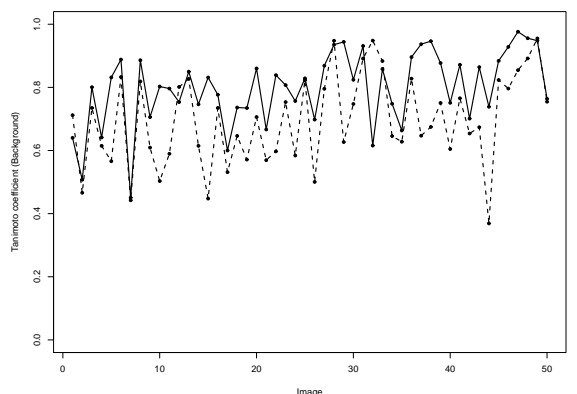

(c)

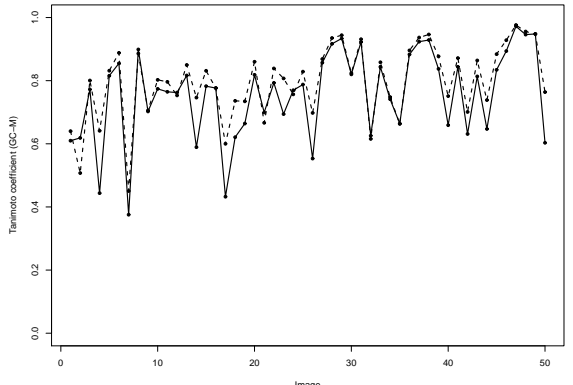

(b)

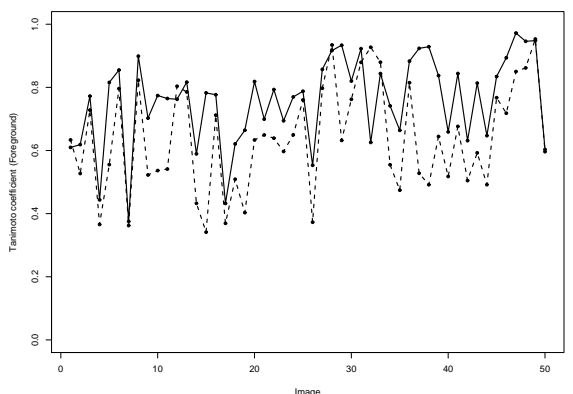

(d)

Fig. 8. Tanimoto coefficient for the supervised pixel classification on the 50 images of [3]. Image order is the same as in Table 4. (a) Background (dashed line) and foreground (solid line) for the I-M. (b) Background (dashed line) and foreground (solid line) for the GC-M. (c) I-M (dashed line) and GC-M (solid line) for the background class. (d) I-M (dashed line) and GC-M (solid line) for the foreground class. 
Table 1 also shows information about the standard deviations for each evaluation measure. For all cases, the standard deviation indicates that using a GC-M in pixel classification is more consistent than using an I-M.

In order to statistically compare the performance of the probabilistic models, Table 2 shows confidence intervals and p-values that confirm differences between the models. None of confidence intervals include the 0 value and all p-values are less than $\alpha=0.05$.

\section{Conclusions}

In this work we introduce the use of Gaussian copulas in supervised pixel classification. According to numerical experiments the selection of a Gaussian copula for modeling structure dependence can help achieve better classification results. An specific example is the image 227092, which appears in Figure 5, its accuracy for the I-M classifier is $57.1 \%$, whereas its accuracy for the GC-M classifier is $89.5 \%$. For this image, the Gaussian copula improves its accuracy.

Although we model the dependence structure for each image with the same copula function, this is not necessary. There are many copula functions and the Gaussian copula has been chosen due to its practical usefulness and easy implementation. However, having more than one copula at hand may improve the performance of the copula-based classifier. In such case, a copula selection procedure is necessary. The evaluation results are the consequence of the selected dependence structure and marginals. For instance, on the image 106024, Figure 4, the performance of the I-M classifier is $57.6 \%$ accurate (accuracy), whereas the GC-M classifier is $58.7 \%$ accurate. For most applications better results can be obtained by selecting the best fitted copula function from a set of available copulas. For example, in the experiment reported, the performance of the I-M classifier is better than GC-M for image fullmoon, Figure 6. However, the GC-M is expected to improve the performance of the I-M classifier if we used the proper copula.

Acknowledgments. The first author acknowledges support from the National Council of Science and Technology of México (CONACyT) through a scholarship to pursue graduate studies in the Department of Computer Science at the Center for Research in Mathematics.

\section{References}

1. Bacigál, T., Komorníková, M.: Fitting Archimedean copulas to bivariate geodetic data. In: Rizzi, A., Vichi, M. (eds.) Compstat 2006 Proceedings in Computational Statistics, pp. 649-656. Physica-Verlag HD, Heidelberg (2006)

2. Bishop, C.M.: Pattern Recognition and Machine Learning. Springer (2007)

3. Blake, A., Rother, C., Brown, M., Perez, P., Torr, P.: Interactive Image Segmentation using an adaptive GMMRF model. In: Proc. European Conference in Computer Vision (ECCV), Springer-Verlag (2004) 
4. Brunel, N., Pieczynski, W., Derrode, S.: Copulas in vectorial hidden Markov chains for multicomponent image segmentation. In: Proceedings of the 2005 IEEE International Conference on Acoustics, Speech and Signal Processing, pp. 717-720 (2005)

5. Cherubini, U., Luciano, E., Vecchiato, W.: Copula Methods in Finance. Wiley, Chichester (2004)

6. De-Waal, D.J., Van-Gelder, P.H.A.J.M.: Modelling of extreme wave heights and periods through copulas. Extremes 8(4), 345-356 (2005)

7. Dowd, K.: Copulas in Macroeconomics. Journal of International and Global Economic Studies, 1(1), 1-26 (2008)

8. Flitti, F., Collet, C., Joannic-Chardin A.: Unsupervised Multiband Image Segmentation using Hidden Markov Quadtree and Copulas. In: IEEE International Conference on Image Processing (2005)

9. Frees, E. W., Valdez, E. A.: Understanding relationships using copulas. North American Actuarial Journal, 2(1), 1-25 (1998)

10. Genest, C., Favre, A.C.: Everything You Always Wanted to Know about Copula Modeling but Were Afraid to Ask. Journal of Hydrologic Engineering, 12(4), 347368 (2007)

11. Grigoriu, M.: Multivariate distributions with specified marginals: Applications to Wind Engineering. Journal of Engineering Mechanics, 133(2), 174-184 (2007)

12. Hastie, T., Tibshirani, R., Friedman, J.: The Elements of Statistical Learning: Data Mining, Inference, and Prediction. Springer (2009)

13. Jajuga, K., Papla, D.: Copula Functions in Model Based Clustering. In: Proceedings of the 29th Annual Conference of the Gesellschaft für Klassifikation e.V., pp. 606-613. Springer (2005)

14. Joe, H.: Multivariate models and dependence concepts. Chapman and Hall, London (1997)

15. Mackay, D.J.C.: Information Theory, Inference, and Learning Algorithms. Cambridge (2008)

16. Mercier, G., Bouchemakh, L., Smara, Y.: The Use of Multidimensional Copulas to Describe Amplitude Distribution of Polarimetric SAR Data. In: IGARSS 07 (2007)

17. Monjardin, P.E.: Análisis de dependencia en tiempo de falla. Master's thesis, Centro de Investigación en Matemáticas. Guanajuato, México (2007) (in Spanish)

18. Nelsen, R. B.: An Introduction to Copulas. Springer, Heidelberg (2006)

19. Sakji-Nsibi, S., Benazza-Benyahia, A.: Multivariate indexing of multichannel images based on the copula theory. In: IPTA08 (2008)

20. Salinas-Gutiérrez, R., Hernández-Aguirre, A., Villa-Diharce, E.R.: Using Copulas in Estimation of Distribution Algorithms. In: MICAI 2009: Advances in Artificial Intelligence, pp. 658-668. Springer (2009)

21. Salinas-Gutiérrez, R., Hernández-Aguirre, A., Villa-Diharce, E.R.: D-vine EDA: a new Estimation of Distribution Algorithm based on Regular Vines. In: Genetic and Evolutionary Conference (GECCO-2010). Accepted for publication (2010)

22. Schölzel, C., Friederichs, P.: Multivariate non-normally distributed random variables in climate research - introduction to the copula approach. Nonlinear Processes in Geophysics, 15(5), 761-772 (2008)

23. Sklar, A.: Fonctions de répartition à $n$ dimensions et leurs marges. Publications de l'Institut de Statistique de l'Université de Paris 8, 229-231 (1959)

24. Stitou, Y., Lasmar, N., Berthoumieu, Y.: Copulas based multivariate gamma modeling for texture classification. In: Proceedings of the 2009 IEEE International Conference on Acoustics, Speech and Signal Processing, pp. 1045-1048 (2009)

25. Trivedi, P.K., Zimmer, D.M.: Copula Modeling: An Introduction for Practitioners. In: vol. 1 of Foundations and Trends ${ }^{\circledR}$ in Econometrics Now Publishers (2007) 
26. Venter, G., Barnett, J., Kreps, R., Major, J.: Multivariate Copulas for Financial Modeling. Variance, 1(1), 103-119 (2007)

27. Image and Video Editing, http://research.microsoft.com/en-us/um/ cambridge/projects/visionimagevideoediting/segmentation/grabcut.htm

\section{Appendix}

Table 3: Description of images used in this work. BG stands for the background class and FG stands for the foreground class. Columns 3 and 4 give the size of test data. The last 4 columns give the number of pixels well classified for each class and for each probabilistic classifier.

\begin{tabular}{|c|c|c|c|c|c|c|c|}
\hline Image name & Image size & Test l & pixels & $\overline{\mathrm{I}-\mathrm{I}}$ & $\bar{M}$ & $\overline{\mathrm{GC}}$ & -M \\
\hline & & BG & FG & BG & FG & BG & FG \\
\hline 21077 & $321 \times 481$ & 4322 & 3353 & 3648 & 2551 & 3144 & 2763 \\
\hline 24077 & $321 \times 481$ & 11529 & 10983 & 6577 & 8399 & 6348 & 10000 \\
\hline 37073 & $321 \times 481$ & 8260 & 6642 & 6404 & 6187 & 7115 & 6014 \\
\hline 65019 & $321 \times 481$ & 9853 & 8398 & 9181 & 3317 & 9099 & 4061 \\
\hline 69020 & $321 \times 481$ & 25203 & 22634 & 17561 & 16813 & 22798 & 20421 \\
\hline 86016 & $321 \times 481$ & 3271 & 2215 & 2765 & 2166 & 2937 & 2179 \\
\hline 106024 & $321 \times 481$ & 9093 & 7368 & 5528 & 3961 & 5574 & 4087 \\
\hline 124080 & $321 \times 481$ & 18286 & 18773 & 16487 & 16924 & 16307 & 18653 \\
\hline 153077 & $321 \times 481$ & 13851 & 12098 & 11072 & 7774 & 10806 & 10638 \\
\hline 153093 & $321 \times 481$ & 12027 & 11809 & 7617 & 8699 & 11414 & 9615 \\
\hline 181079 & $481 \times 321$ & 23845 & 23110 & 18650 & 15320 & 22494 & 18705 \\
\hline 189080 & $481 \times 321$ & 23363 & 23523 & 20726 & 21020 & 19722 & 20707 \\
\hline 208001 & $481 \times 321$ & 10227 & 9530 & 9994 & 7669 & 10064 & 7914 \\
\hline 209070 & $321 \times 481$ & 6696 & 4075 & 5117 & 2447 & 5894 & 2874 \\
\hline 227092 & $481 \times 321$ & 19656 & 17321 & 12869 & 8229 & 19129 & 13966 \\
\hline 271008 & $321 \times 481$ & 10909 & 9216 & 8934 & 7967 & 8800 & 8795 \\
\hline 304074 & $481 \times 321$ & 7239 & 4794 & 5017 & 2591 & 5534 & 2810 \\
\hline 326038 & $321 \times 481$ & 10781 & 7680 & 8730 & 4952 & 9488 & 5571 \\
\hline 376043 & $481 \times 321$ & 13654 & 13485 & 12022 & 6094 & 13072 & 9343 \\
\hline 388016 & $481 \times 321$ & 17800 & 15592 & 15633 & 11248 & 17596 & 12929 \\
\hline banana1 & $480 \times 640$ & 29983 & 24052 & 17120 & 23964 & 20285 & 23601 \\
\hline banana 2 & $480 \times 640$ & 27433 & 21518 & 17063 & 20378 & 25373 & 18698 \\
\hline banana3 & $480 \times 640$ & 26205 & 20164 & 25588 & 12405 & 26035 & $\mid 14115$ \\
\hline book & $480 \times 640$ & 26087 & 21474 & 15689 & 20699 & 19852 & 21325 \\
\hline bool & $450 \times 520$ & 20123 & 16850 & 19500 & 13279 & 18726 & 14373 \\
\hline bush & $600 \times 450$ & 32513 & 22099 & 21072 & 12504 & 27734 & 14870 \\
\hline ceramic & $480 \times 640$ & 30549 & 25709 & 24809 & 25069 & 27328 & 24791 \\
\hline cross & $600 \times 450$ & 34602 & 25733 & 32824 & 25703 & 32918 & 25132 \\
\hline doll & $549 \times 462$ & 18866 & 15106 & 12976 & 13269 & 17947 & 14960 \\
\hline elefant & $480 \times 640$ & 27858 & 22787 & 20918 & 22656 & 23158 & $22540 \mid$ \\
\hline flower & $450 \times 600$ & 16125 & 13246 & 14612 & 12977 & 15036 & 13225 \\
\hline fullmoon & $350 \times 442$ & 1580 & 1043 & 1498 & 1043 & 983 & 1026 \\
\hline grave & $600 \times 450$ & 12294 & 12832 & 11977 & 11567 & 12219 & 10889 \\
\hline
\end{tabular}


Table 3 - continued from previous page

\begin{tabular}{|c|r|r|r|r|r|r|r|}
\hline Image name & Image size & \multicolumn{1}{|c|}{ Test pixels } & \multicolumn{2}{|c|}{ I-M } & \multicolumn{2}{|c|}{ GC-M } \\
\hline & & \multicolumn{1}{|c|}{ BG } & \multicolumn{1}{|c|}{ FG } & \multicolumn{1}{|c|}{ BG } & \multicolumn{1}{|c|}{ FG } & \multicolumn{1}{|c|}{ BG } & \multicolumn{1}{|c|}{ FG } \\
\hline llama & $371 \times 513$ & 8930 & 8445 & 7783 & 5322 & 7547 & 7287 \\
memorial & $600 \times 450$ & 14853 & 12598 & 12900 & 6902 & 10936 & 10964 \\
music & $480 \times 640$ & 23945 & 19494 & 20457 & 18723 & 21794 & 19112 \\
person1 & $450 \times 600$ & 19092 & 16384 & 16452 & 10041 & 18831 & 15372 \\
person2 & $450 \times 600$ & 12796 & 9595 & 11492 & 5358 & 12465 & 9219 \\
person3 & $600 \times 450$ & 14649 & 11450 & 13494 & 8122 & 14022 & 10112 \\
person4 & $450 \times 600$ & 19250 & 16631 & 15230 & 10691 & 18197 & 11653 \\
person5 & $600 \times 450$ & 13990 & 11332 & 13009 & 8327 & 13025 & 10377 \\
person6 & $600 \times 450$ & 19015 & 15645 & 16753 & 9038 & 16071 & 11732 \\
person7 & $600 \times 450$ & 12110 & 9634 & 9934 & 6998 & 11795 & 8093 \\
person8 & $480 \times 640$ & 16684 & 12741 & 6740 & 11157 & 14690 & 9534 \\
scissors & $480 \times 640$ & 30768 & 23335 & 28152 & 19910 & 30181 & 19960 \\
sheep & $600 \times 450$ & 5331 & 3733 & 4750 & 3098 & 5243 & 3415 \\
stone1 & $480 \times 640$ & 18716 & 15635 & 16087 & 15525 & 18376 & 15528 \\
stone2 & $480 \times 640$ & 22002 & 18489 & 21556 & 16315 & 21788 & 17692 \\
teddy & $398 \times 284$ & 13892 & 13739 & 13790 & 13191 & 13426 & 13466 \\
tennis & $472 \times 500$ & 19471 & 13129 & 18054 & 8673 & 18322 & 8613 \\
\hline \hline
\end{tabular}

Table 4: Evaluation measures for each image. TC stands for the Tanimoto coefficient, BG stands for the background class and FG stands for the foreground class. Columns 2, 3 and 4 give the results for the independent probabilistic model. The last 3 columns give the results for the Gaussian copula-based probabilistic model.

\begin{tabular}{|c|r|r|r|r|r|r|}
\hline Image name & \multicolumn{3}{|c|}{ I-M } & \multicolumn{3}{|c|}{ GC-M } \\
\hline & TC-BG & TC-FG & Accuracy & TC-BG & TC-FG & Accuracy \\
\hline 21077 & 0.712 & 0.633 & 0.808 & 0.640 & 0.610 & 0.770 \\
24077 & 0.466 & 0.527 & 0.665 & 0.507 & 0.619 & 0.726 \\
37073 & 0.735 & 0.728 & 0.845 & 0.801 & 0.772 & 0.881 \\
65019 & 0.615 & 0.366 & 0.685 & 0.641 & 0.444 & 0.721 \\
69020 & 0.566 & 0.555 & 0.719 & 0.832 & 0.816 & 0.903 \\
86016 & 0.833 & 0.796 & 0.899 & 0.888 & 0.855 & 0.933 \\
106024 & 0.442 & 0.362 & 0.576 & 0.450 & 0.375 & 0.587 \\
124080 & 0.819 & 0.823 & 0.902 & 0.886 & 0.899 & 0.943 \\
153077 & 0.609 & 0.523 & 0.726 & 0.706 & 0.703 & 0.826 \\
153093 & 0.503 & 0.536 & 0.685 & 0.803 & 0.774 & 0.882 \\
181079 & 0.590 & 0.541 & 0.723 & 0.796 & 0.765 & 0.877 \\
189080 & 0.801 & 0.804 & 0.890 & 0.753 & 0.762 & 0.862 \\
208001 & 0.827 & 0.786 & 0.894 & 0.850 & 0.816 & 0.910 \\
209070 & 0.615 & 0.433 & 0.702 & 0.746 & 0.589 & 0.814 \\
227092 & 0.448 & 0.341 & 0.571 & 0.831 & 0.782 & 0.895 \\
271008 & 0.735 & 0.712 & 0.840 & 0.777 & 0.777 & 0.874 \\
304074 & 0.531 & 0.369 & 0.632 & 0.600 & 0.432 & 0.693 \\
\hline & \multicolumn{7}{|c|}{ Continued on next page } \\
\hline
\end{tabular}


Table 4 - continued from previous page

\begin{tabular}{|c|r|r|r|r|r|r|}
\hline Image name & \multicolumn{3}{|c|}{ I-M } & \multicolumn{3}{|c|}{ GC-M } \\
\hline & TC-BG & TC-FG & Accuracy & TC-BG & TC-FG & Accuracy \\
\hline 326038 & 0.646 & 0.509 & 0.741 & 0.736 & 0.621 & 0.816 \\
376043 & 0.571 & 0.403 & 0.668 & 0.735 & 0.664 & 0.826 \\
388016 & 0.706 & 0.633 & 0.805 & 0.860 & 0.818 & 0.914 \\
banana1 & 0.569 & 0.649 & 0.760 & 0.667 & 0.699 & 0.812 \\
banana2 & 0.597 & 0.639 & 0.765 & 0.839 & 0.793 & 0.900 \\
banana3 & 0.753 & 0.597 & 0.819 & 0.807 & 0.694 & 0.866 \\
book & 0.584 & 0.649 & 0.765 & 0.757 & 0.770 & 0.866 \\
bool & 0.823 & 0.760 & 0.887 & 0.829 & 0.788 & 0.895 \\
bush & 0.500 & 0.373 & 0.615 & 0.698 & 0.553 & 0.780 \\
ceramic & 0.795 & 0.797 & 0.887 & 0.868 & 0.857 & 0.926 \\
cross & 0.948 & 0.934 & 0.970 & 0.935 & 0.917 & 0.962 \\
doll & 0.627 & 0.632 & 0.773 & 0.944 & 0.934 & 0.969 \\
elefant & 0.747 & 0.762 & 0.860 & 0.824 & 0.820 & 0.902 \\
flower & 0.891 & 0.879 & 0.939 & 0.931 & 0.923 & 0.962 \\
fullmoon & 0.948 & 0.927 & 0.969 & 0.616 & 0.626 & 0.766 \\
grave & 0.883 & 0.880 & 0.937 & 0.858 & 0.844 & 0.920 \\
llama & 0.646 & 0.555 & 0.754 & 0.748 & 0.741 & 0.854 \\
memorial & 0.628 & 0.474 & 0.721 & 0.663 & 0.664 & 0.798 \\
music & 0.828 & 0.815 & 0.902 & 0.896 & 0.883 & 0.942 \\
person1 & 0.647 & 0.528 & 0.747 & 0.937 & 0.924 & 0.964 \\
person2 & 0.675 & 0.492 & 0.753 & 0.946 & 0.929 & 0.968 \\
person3 & 0.751 & 0.644 & 0.828 & 0.877 & 0.837 & 0.925 \\
person4 & 0.605 & 0.518 & 0.722 & 0.751 & 0.659 & 0.832 \\
person5 & 0.765 & 0.676 & 0.843 & 0.872 & 0.844 & 0.924 \\
person6 & 0.654 & 0.505 & 0.744 & 0.701 & 0.631 & 0.802 \\
person7 & 0.674 & 0.593 & 0.779 & 0.864 & 0.813 & 0.915 \\
person8 & 0.369 & 0.492 & 0.608 & 0.739 & 0.647 & 0.823 \\
scissors & 0.823 & 0.767 & 0.888 & 0.884 & 0.834 & 0.927 \\
sheep & 0.796 & 0.718 & 0.866 & 0.928 & 0.894 & 0.955 \\
stone1 & 0.855 & 0.850 & 0.920 & 0.976 & 0.972 & 0.987 \\
stone2 & 0.892 & 0.862 & 0.935 & 0.956 & 0.946 & 0.975 \\
teddy & 0.955 & 0.953 & 0.976 & 0.948 & 0.948 & 0.973 \\
tennis & 0.755 & 0.596 & 0.820 & 0.764 & 0.603 & 0.826 \\
\hline \hline & & & & & & \\
\hline
\end{tabular}

